\title{
Bias Flow Acoustic Liner with Straight and Tapered Apertures
}

\author{
Soufiane Ramdani ${ }^{1}$, Nobuhiko Yamasaki ${ }^{1}$, Yuzo Inokuchi ${ }^{2}$, Tatsuya Ishii ${ }^{3}$ \\ ${ }^{1}$ Department of Aeronautics and Astronautics \\ Kyushu University \\ 744 Motooka, Nishi-ku, Fukuoka 819-0395, JAPAN \\ ${ }^{2}$ Civil Aviation College \\ ${ }^{3}$ Japan Aerospace Exploration Agency
}

\begin{abstract}
Experimental and computational studies are performed on slit resonators, i.e., one aperture with a high aspect ratio that spans through the center of the plate.

An impedance tube experiment is conducted to investigate the macroscopic response of slit apertures. The test specimens include straight and tapered apertures. Subsequently, the effect of introducing a bias flow is investigated.

The absorption performance increases when the sound pressure level increases to a level that causes a production of shed vortices. When a bias flow is introduced, the absorption coefficient reaches its maximum absorption of the incident sound wave in the region near the resonant frequency for a Mach number close to $9.71 \times 10^{-3}$

2D numerical simulations are performed and validated with the experimental results. Good agreement is obtained for the majority of the simulated cases. Vortex shedding and its effect on the absorption coefficients is also investigated.
\end{abstract}

\section{INTRODUCTION}

Acoustic liners are widely used devices in aero-engines, and they are installed on the inner side of the nacelle to reduce fan noise in turbofan engines of commercial airplanes (conventional acoustic liners without bias flow). They are also used in the combustion chamber to reduce the acoustic instabilities caused by the combustion (acoustic liners with bias flow where the bias flow is caused by secondary air flow). An acoustic liner is typically made of a perforated metal sheet backed by a cavity. Each aperture of the perforated sheet and the cavity form a Helmholtz resonator. The resonator effectively absorbs the sound near the resonant frequency, however, its absorbing performance decreases at off-resonant frequencies.

Howe [1] theoretically proposed that a low frequency (low Strouhal number) sound wave can be significantly attenuated by a jet flow by converting the acoustical energy into energy of fluctuating vorticity, which is shed from the nozzle edge. Bechert [2] proposed another theory to explain this phenomenon, and this was supported via experimental data. Bechert [2] also proposed a simple theory to predict the optimum Mach number of bias flow to obtain the perfect attenuation. On the other hand, Howe's theory to predict the sound absorption coefficient including the effects of a bias flow is well supported by an experiment by Hughes and Dowling [3]. Hence, this led to the idea that the off-resonant performance of a resonator can be improved if a jet (or a bias flow) is introduced from an aperture of an acoustic liner. Lahiri et al. [4] collected this type of experimental data and showed that the application of a bias flow through the aperture widens the frequency range of dissipation, with the penalty of reduced peak performance near the resonant frequency. Zhao and Li [5] wrote a summary on tunable acoustic liners including a liner with bias flow.

In the field of numerical simulation, Mendez and Eldredge [6] performed a 3D large eddy simulation (LES), and Ji and Zhao [7] performed a 2D lattice Boltzman method (LBM) for an aperture with bias flow, and both made a comparison with Howe's theory, obtaining good agreements. Roche et al. [8] conducted 3D and 2D axisymmetric numerical simulations for a cylindrical acoustic resonator in the case without a bias flow using direct numerical simulation (DNS). Good agreement was obtained between 2D and 3D results. In a previous study (Ramdani et al. [9]), 2D simulations of a slit resonator were conducted using LES for cases with and without bias flow. Good agreement was obtained for the non-bias flow case. However, the bias flow case was not validated due to the lack of the experimental results for the simulated model. Tam et al. [10] conducted a series of experiments and DNS simulations to slit apertures with a $90^{\circ}$ corner (straight aperture) and $45^{\circ}$ corner (tapered aperture). The results obtained using the simulation supports the usage of computational aeroacoustics (CAA) as a design tool.

Wada and Ishii [11] performed experiments for acoustic liners with a bias flow passing through the apertures of a perforated plate (circular straight perforations) and observed that the absorption range of the liner became wider and was not concentrated around the resonant frequency as in the case of the conventional liner. They compared the experimental results with Howe's extended theory proposed by Luong et al. [12], which considered the thickness of the perforated sheet and obtained good agreements. In a previous study (Tanaka et al. [13]), the macroscopic effect of the design parameters (such as the shape of the aperture and the flow velocity when a bias flow is applied through the aperture) on the impedance of an acoustic resonator was experimentally investigated via an acoustic impedance tube. The results revealed that the fully tapered aperture exhibited a wider absorption frequency range when compared to that of a straight circular aperture. However, little was known about the reason for such a behavior given the difficulty of visualizing the flow around the small apertures in the experimental setup using the impedance tube.

In the present study, the acoustic performance of the liner and the flow field around the perforated plate is numerically solved using the compressible Navier-Stokes equations to understand the acoustic and fluid dynamic behavior of the liner and the effect of the shape of the perforation at a microscopic level.

Manuscript Received on October 17, 2018

Review Completed on June 27, 2019 


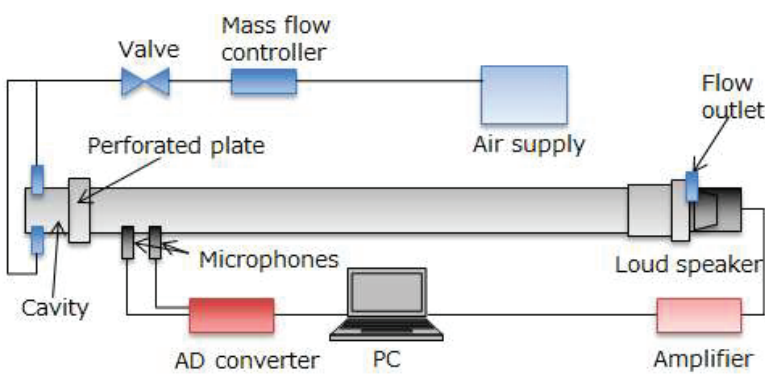

Fig. 1 Experimental setup
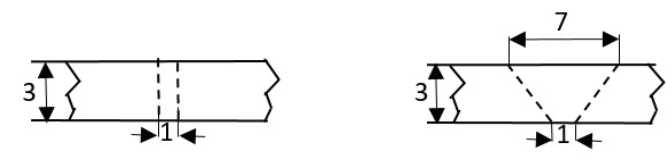

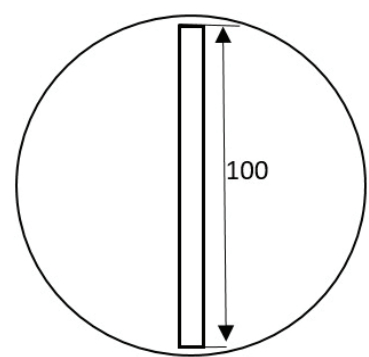
unit: $\lceil\mathrm{mm}\rceil$
Fig. 2 Straight aperture,

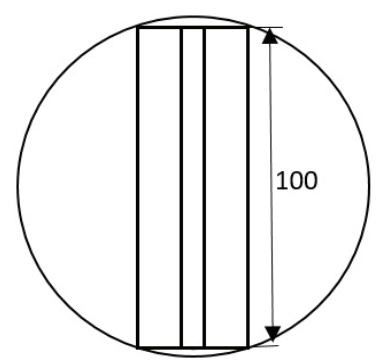

Fig. 3 Tapered aperture, unit: $[\mathrm{mm}]$
The aforementioned studies involve a long computational time and computational resources to perform 3D simulations. Thus, in the present study, the simulations are conducted using 2D large eddy simulations. For the 2D assumption to be acceptable, the impedance tube experiment is conducted on slit apertures where slit indicates that each plate has only one aperture with a high aspect ratio of 100 . The aperture spans through the center of the plate. Subsequently, numerical simulations are conducted to focus on the effect of bias flow on the absorption performance of the acoustic liner and flow field around the apertures.

\section{EXPERIMENTAL CONFIGURATION}

The impedance tube experimental rig used is shown in Fig. 1. The internal diameter of the tube is $100 \mathrm{~mm}$ and it has a length of 2 m.

A full-range speaker (FF85WK, FOSTEX) is attached at an end of the tube to generate sound waves. At the other end of the tube, the resonator specimen under test is attached. The cavity of the resonator has a depth of $52 \mathrm{~mm}$ and it has an internal diameter of 100 $\mathrm{mm}$. The diameter makes it possible to examine low-frequency acoustic resonators since it exhibits a cut-off frequency of approximately $2000 \mathrm{~Hz}$. Two different perforated plates made of aluminum with a thickness of $3 \mathrm{~mm}$ with different aperture geometry are installed between the cavity section and tube section. Such thick plates are selected to stiffen the plates to reduce the absorption caused by the vibration of the plates.

In order to produce a bias flow through the perforated plate, two air supply holes are created on the cavity side wall and are connected to compressed air by using flexible pipes. A mass flow controller (KOFLOC 8550) is used to regulate the desired flow rate. The flow outlet is adjacent to the loud speaker.

The white noise is generated by the computer and fed to an amplifier (INTEGRATED AMPLIFIER PM4200, Marantz) before it is finally fed to the loudspeaker.

Two microphones (GRAS 40PH) are attached to the impedance tube at distances corresponding to $148 \mathrm{~mm}$ and $218 \mathrm{~mm}$ from the face of the perforated plate of the resonator. Both microphones are attached to an AD recorder (TEAC LX-110) and the recorded data is stored in a computer. For each case, ten samples with a data length of 600000 and sampling rate of $12 \mathrm{kHz}$ are recorded.

The experiment is conducted at atmospheric conditions, with a temperature of approximately $13{ }^{\circ} \mathrm{C}$ and a sea level atmospheric pressure of $101.3 \mathrm{kPa}$.

Figures 2 and 3 show the geometry of the two plates with the slit apertures used. The diameter of the plates corresponds to 110 $\mathrm{mm}$ with a thickness of $h=3 \mathrm{~mm}$. Each plate has only one slit aperture spanning through the center. The straight aperture in Fig. 2 exhibits a cuboid with a width of $b=1 \mathrm{~mm}$ and length of $100 \mathrm{~mm}$. The tapered aperture in Fig. 3 exhibits a trapezoidal shape with a width of $7 \mathrm{~mm}$ at the base and $1 \mathrm{~mm}$ at the top. Both plates have an open area ratio of $P=1.27 \%$. The open area ratio is defined as the ratio of the area of the aperture and area of the impedance tube.

The resonant frequency of a resonator is related to its aperture thickness $h$, cavity depth $l$, and open area ratio $P$ and is given by Eq. (1) as follows:

$$
f_{0}=\frac{c}{2 \pi} \sqrt{\frac{P}{l(h+\delta)}}
$$

where $\delta$ denotes the end correction and $c$ denoted the speed of sound.

For a straight slit resonator, the end correction is estimated by Eq. (2) as follows:

$$
\delta=K b ; K=-\frac{2}{\pi} \ln \left[\sin \left(\frac{\pi}{2}\right)\right]
$$

Subsequently, the estimated resonant frequency of the straight slit aperture is $365 \mathrm{~Hz}$.

The results of the experiments discussed in a later section indicate that the resonant frequency for the straight aperture corresponds to approximately $340 \mathrm{~Hz}$ while the resonant frequency for the tapered aperture corresponds to approximately $460 \mathrm{~Hz}$.

\section{NUMERICAL CONFIGURATION}

In this study, large eddy simulation (LES) is conducted by using the numerical code UPACS-LES developed by Japan Aerospace Exploration Agency (JAXA). Compressible Navier-Stokes equations are solved as follows:

$$
\frac{\partial \boldsymbol{Q}}{\partial t}+\frac{\partial \boldsymbol{F}_{j}^{C}}{\partial x_{j}}+\frac{\partial \boldsymbol{F}_{j}^{V}}{\partial x_{j}}=\boldsymbol{S}
$$

where

$\boldsymbol{Q} \quad$ : Conservative variables vector composed of density, momentum vector, and energy

$\boldsymbol{F}_{j}^{C} \quad$ : Convective term of Navier-Stokes equations

$\boldsymbol{F}_{j}^{V} \quad$ : Diffusive term of Navier-Stokes equations

The source term vector $\boldsymbol{S}$ of Navier-Stokes equations is denoted as shown in Eq. (4). In the present formulation, the incoming sound waves and bias flows are introduced as sources instead of using the conventional boundary conditions to stipulate the incoming sound waves and bias flows. Thus, the source term $\boldsymbol{S}$ has a finite value in specified regions where the sound source and the mass source are implemented.

$$
\boldsymbol{S}=\left[S_{1}, S_{2}, S_{3}, S_{4}, S_{5}\right]^{\mathrm{T}}
$$

The UPACS-LES code numerically solves Eq. (3) via the finite volume method (FVM) for the multi-block structured mesh. The implicit large eddy simulation (ILES) approach is used to numerically simulate a 2 -D slit acoustic liner. 


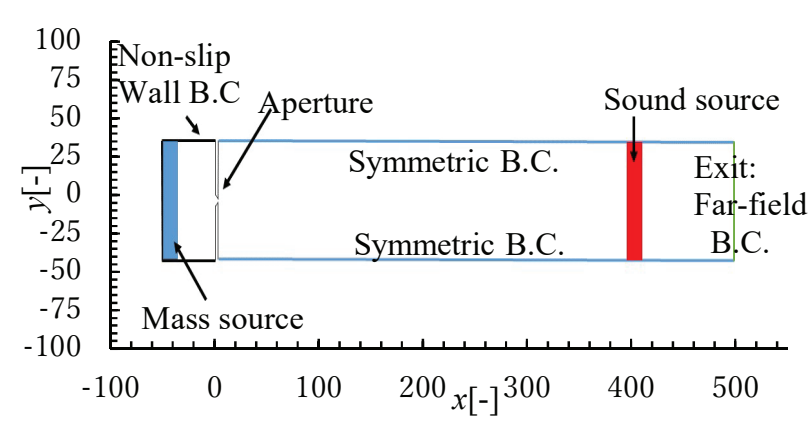

Fig. 4 Sources locations and boundary conditions

The resonant frequency in a Helmholtz resonator is estimated by Eq. (1). In order for the geometry of the slit resonator in the $2 \mathrm{D}$ simulations to exhibit resonant frequencies close to the experimental ones, open area ratio $P$, plate thickness $h$, and cavity depth $l$ are maintained identical. This leads to a constant cavity area to slit area ratio equal to $d=78.5$.

It should be noted that the configuration of the numerical simulations neglects the three-dimensionality effects, and thus this corresponds to an approximation since it assumes infinitely long slits spaced at constant distances of $d$ while the experimental plates are of circular shape as shown in Figs. 2 and 3.

Figure 4 shows the geometry and dimensions of the slit resonator analyzed. The length of the impedance tube is set to $500 \mathrm{D}$. The width of the aperture is $D=1 \mathrm{~mm}$ and the thickness of the perforated plate is $h=3 D$ and it contains slits of dimensions shown in Figs. 2 and 3. The cavity has a depth of $l=52 D$ and a width of $78.5 \mathrm{D}$.

The non-dimensionalized time $t$, coordinate variables $x_{i}$, velocities $u_{i}$, pressure $p$, and vorticity $\Omega$ are calculated as follows:

$$
t=\frac{t^{*}}{D / c}, x_{i}=\frac{x_{i}^{*}}{D}, u_{i}=\frac{u^{*}}{c}, p=\frac{p^{*}}{\rho c^{2}}, \Omega=\frac{\Omega^{*}}{c / D}
$$

The calculation domain consists of 36 blocks with a higher resolution in the blocks close to the aperture. The resolution is lower for the blocks away from it. This is because the length scale at a distance from the aperture is in the range of the wavelength. However, near the aperture, an oscillatory viscous wall layer called the Stokes layer is formed, and it should be captured. The Stokes layer exhibits a length scale that is extremely low when compared to the wavelength.

The grid spacing in the aperture corresponds to $D / 79$ in the $y$ direction and $h / 79$ in the $x$-direction. This resolution can resolve the Stokes layer and the viscous sublayer of the boundary layer with a dimensionless wall distance $\left(y^{+}\right)$that corresponds to 1 .

The number of cells in the z-direction corresponds to one with the symmetric boundary condition applied on both sides.

The non-dimensional time step is selected as $\Delta t=3.15 \times$ $10^{-3}$, and leads to a CFL number lower than 1.

Room temperature of $293.15 \mathrm{~K}$ and atmospheric pressure of $101.3 \mathrm{kPa}$ are given as initial conditions for the numerical simulations. Given these conditions, density is $\rho=1.2 \mathrm{~kg} / \mathrm{m}^{3}$ and speed of sound is $c=343.2 \mathrm{~m} / \mathrm{s}$. The absorption coefficient for each case is calculated with data length equivalent to 10 incident waves such that each wave is captured by 20 data points.

The 6th-order compact scheme with the 10th-order compact filter is used to solve the convective terms of the conservation equations, while the 2nd-order central discretization is used for the viscous terms.

The obtained space discretized equations are then integrated in time via the 3rd-order Runge-Kutta explicit scheme.

The model analyzed in the study is shown in Fig. 4 and represents the case of the perforated plate backed by a cavity with a mass source region that can be activated to introduce a bias flow passing through the aperture.

The geometry of the model and applied boundary conditions are shown in Fig. 4. It contains an outlet with a far-field boundary condition, which corresponds to a non-reflective boundary condition using the characteristic waves and the inner points to determine the boundary conditions. The cavity is backed by a non-slip wall boundary condition. Symmetric boundary conditions are applied to the sides, and non-slip walls are applied to the perforated plate. Additionally, a sound source is applied at a distance of $400 \mathrm{D}$ from the aperture.

When simulating the bias flow case, first, the bias flow jet is fully developed. The simulation is run for 72 hours and it requires about 1.5 million iterations. The convergence is verified by checking the convergence of the residuals and also by verifying the value of the bias flow at the aperture which should be equal to the desired bias flow. Then, the sound wave source is enabled. The time required for three waves to propagate is found to be enough for all the frequencies simulated before starting the sampling process.

The simulations are conducted for frequencies from $200 \mathrm{~Hz}$ to $700 \mathrm{~Hz}$ for both tested plates and with sound pressure level (SPL) values corresponding to $100 \mathrm{~dB}$ and $115 \mathrm{~dB}$ for both plates.

The results indicated that the SPL significantly affects the absorption performance for both the experiment and simulation. Therefore, we tested two different SPLs of $100 \mathrm{~dB}$ and $115 \mathrm{~dB}$. The reason for the dependency on SPL is discussed in the subsequent sections.

The sound source is implemented in the solver as given in Eqs. (6), (7) and (8).

$$
\begin{gathered}
S_{1}=\frac{\rho A u_{a c}(t)}{V_{s s}} \\
S_{2}=S_{1} \times u_{a c}(t) \\
S_{5}=S_{1}\left(C_{v} T_{0}+\frac{1}{2}\left(u_{a c}(t)\right)^{2}\right)
\end{gathered}
$$

where

$V_{S S} \quad:$ Volume of the sound source region

$u_{a c} \quad:$ Acoustic particle velocity

$C_{v} \quad:$ Specific heat at constant volume

$T_{0} \quad$ : Temperature

with an acoustic particle velocity changing sinusoidally in time as given in Eq. (9)

$$
u_{a c}(t)=u_{a} \sin (\omega t)
$$

Here $\omega$ denotes the angular frequency and $u_{a}$ denotes the amplitude of the acoustic particle velocity that leads to the amplitude of the specified acoustic pressure wave $p_{a}$.

The amplitude of the acoustic particle velocity is calculated as $u_{a}=p_{a} / Z_{0}$, where $Z_{0}=\rho c$ denotes the characteristic impedance of the air.

Equations 6, 7 and 8 create left and right going waves. The right going waves exit the impedance tube without being reflected and thus have no influence on the resonator. However, the left going waves create a standing wave between the source region and the perforated wall of the resonator. The standing wave is then used to determine the performance of the resonator.

In order to add a bias flow exiting the cavity of the resonator in the direction of the acoustic tube and to simultaneously simulate the reflection of the sound wave from the back wall of the cavity, a mass source is added in Eq. (3) in the region near the back plate. In order to realize this, in addition to defining the mass source $S_{1}$ (Eq. (10)), it is necessary to add momentum source in the $x$-direction (Eq. (11)) and energy source $S_{5}$ (Eq. (12)) as follows: 


$$
\begin{gathered}
S_{1}=\frac{\dot{m}_{\text {total }}}{V_{\text {total }}} \\
S_{2}=\frac{\dot{m}_{\text {total }} u_{\text {jet }}}{V_{\text {total }}} \\
S_{5}=\dot{m}_{\text {total }}\left(C_{v} T_{0}+\frac{1}{2}\left(u_{\text {jet }}\right)^{2}\right) \frac{1}{V_{\text {total }}}
\end{gathered}
$$

$\begin{array}{ll}\text { where } & \\ \dot{m}_{\text {total }} & : \text { Required mass flow } \\ V_{\text {total }} & : \text { Volume of the mass source region } \\ u_{\text {jet }} & : \text { Velocity at the aperture }\end{array}$

The transfer function method (ISO 10534-2 [14]) is the procedure that is used to measure the acoustic impedance and thereby the absorption coefficients of the experimentally tested and simulated plates. It is also referred to as the two-microphone method. Figure 1 shows a normal incidence acoustic impedance tube in which two microphones are fixed.

The absorption coefficient $\alpha$ is calculated as the fraction of the sound power absorbed in the liner to the incident sound power, and it is expressed as the ratio of the absorbed sound intensity $I_{a b s}$ to the incident sound intensity $I_{i n c}$ as follows:

$$
\alpha=\frac{I_{a b s}}{I_{\text {inc }}}
$$

Viscous effects cause the absorption of acoustic waves in perforated plates (Tam et al. [15] and Zhang et al. [16]). Thus, it is important to calculate the energy rate at which acoustic energy is converted to viscous dissipation as given in Eq. (14).

$$
E_{\text {viscous }}^{\mathrm{v}}=\iiint_{V} \bar{D}(x, y, z) d x d y d z
$$

Equation (14) corresponds to the integration of the timeaveraged dissipation rate $\bar{D}(x, y)$ given in Eq. (15) over a domain $V$ large enough to include the shed vortices from the aperture.

$$
\bar{D}(x, y, z)=\frac{1}{T} \int_{0}^{T} \Phi d t
$$

In Eq. (16), $\Phi$ is called the viscous dissipation function, and it denotes the rate of conversion of the viscous force work to internal energy. The viscous dissipation function is expressed for a Newtonian fluid as specified by Greitzer et al. [17].

$$
\begin{aligned}
\Phi= & \tau_{i j} \frac{\partial u_{i}}{\partial x_{j}}=\underbrace{2 \mu\left[\left(\frac{\partial u}{\partial x}\right)^{2}+\left(\frac{\partial v}{\partial y}\right)^{2}+\left(\frac{\partial w}{\partial z}\right)^{2}\right]}_{\Phi_{1}} \\
& +\underbrace{2 \mu\left[-\frac{1}{3}\left(\frac{\partial u}{\partial x}+\frac{\partial v}{\partial y}+\frac{\partial w}{\partial z}\right)^{2}\right]}_{\Phi_{3}} \\
+ & \underbrace{\mu\left[\left[\frac{\partial v}{\partial x}+\frac{\partial u}{\partial y}\right]^{2}+\left[\frac{\partial w}{\partial y}+\frac{\partial v}{\partial z}\right]^{2}+\left[\frac{\partial u}{\partial z}+\frac{\partial w}{\partial x}\right]^{2}\right]}_{\Phi_{2}}
\end{aligned}
$$

The viscosity of air $\mu$ is calculated via Sutherland's law. Eq. (16) corresponds to the sum of three terms. $\Phi_{1}$ denotes the dissipation caused by the compressive changes of the fluid element. $\Phi_{2}$ is the dissipation caused by the dilatation of the fluid element. And finally, $\Phi_{3}$ accounts for the dissipation caused by angular deformation when the considered fluid element undergoes shear deformation.

In a previous study [9], the sound absorption coefficient was evaluated via the viscous dissipation method, in which $E_{\mathrm{viscous}}^{\mathrm{v}}(\mathrm{Eq}$
14 ) is used, as well as by using the two microphone method. The absorption coefficients obtained by these two methods agree well when there is no bias flow, however, the viscous dissipation method can't be used when a bias flow is introduced. By definition, the viscous dissipation method assumes that all the viscous dissipation is caused by the sound wave. Hence, in this study, the comparison of the absorption coefficient between the experiments and the simulations is performed via the two microphone method. The viscous dissipation method is used to evaluate the spatial distributions of dissipation between the straight and tapered aperture in the cases without bias flow. A previous study [9] indicated that the most dominant dissipation component in cases without bias flow corresponds to $\Phi_{3}$ and the contribution from $\Phi_{2}$ is negligible.

\section{THEORETICAL MODEL}

A Helmholtz resonator impedance is decomposed into two components, namely the impedance of the aperture and impedance of the cavity.

The total impedance of the resonator is then given by Eq. (17) as follows:

$$
Z_{\mathrm{t}}=Z_{\text {cavity }}+Z_{\text {aperture }}
$$

where the impedance of the cavity is given by Eq. (18) as a function of the wave number $k$ and the cavity depth $l$ as follows:

$$
Z_{\text {cavity }}=-i \cot (k l)
$$

The impedance of the aperture is given by Eq. (19) as follows:

$$
Z_{\text {aperture }}=r+i X
$$

where $r$ and $X$ are the resistance and the reactance of the aperture, respectively.

\section{Conventional slit resonator model}

Ulf and Tor [18] proposed a theoretical model for straight slit apertures in the linear regime. The resistance of the aperture is given by Eq. (20)

$$
r=\frac{1}{2 \rho c} \sqrt{2 \mu \rho \omega}\left(4+\frac{2 h}{b}\right)
$$

where $\rho$ denotes density, $\mu$ denotes the viscosity of air, $\omega$ denotes angular frequency, $h$ denotes the thickness of the plate, and $b$ denotes the width of the aperture.

Additionally, the reactance of the aperture is $X=k(h+\delta)$ where $\delta$ denotes the end correction calculated using Eq. (2)

\section{Bias flow slit resonator model}

Hughes and Dowling [19] investigated a plate with parallel slits, through which a bias flow was introduced. As a result, a theoretical model was obtained for the linear regime. In this section, a brief explanation of the model is given.

The theoretical model assumes an infinitely thin screen with infinitely long slits spaced regularly at a distance $d$ and with an open area ratio $P$.

For a screen with a backing plate, the reflection coefficient is given by Eq. (21) as follows:

$$
R=\frac{2 k d[(\ln 2 / \varphi)-\ln (\pi P)]-(\pi / \tan (k l))-i \pi}{2 k d[(\ln 2 / \varphi)-\ln (\pi P)]-(\pi / \tan (k l))+i \pi}
$$

where $i$ denoted the imaginary unit, $k$ denotes the wave number, $l$ denotes the cavity depth and $\varphi$ is a function of Strouhal number that accounts for the effect of the vortex shedding.

The Strouhal number is defined in Eq (22) as follows: 


$$
S_{t}=\frac{\omega a}{U}
$$

where

$$
\begin{array}{ll}
\omega & : \text { Angular frequency of the sound wave } \\
a & : \text { Half the width of the aperture } \\
U & : \text { Vorticity convection velocity (considered equal to the }
\end{array}
$$$$
\text { bias flow velocity by Hughes and Dowling [3]. }
$$

In the study, a straight slit aperture plate of finite thickness is used. Thus, in order to account for the thickness of the aperture in the theoretical model, the reactance of the aperture thickness $X_{\text {aperture }}$ is given by Eq. (23) for an aperture thickness $h$ and is added to the impedance $Z$ of the thin screen obtained in Eq. (21) by using Eq. (24). The method is similar to that proposed by Jing and Sun [20] for a straight circular aperture.

The total impedance of the perforated plate backed by a cavity is then given by Eq. (25).

$$
\begin{gathered}
X_{\text {aperture }}=-i k \frac{h}{P} \\
Z=\frac{1+R}{1-R} \\
Z_{\mathrm{t}}=Z+X_{\text {aperture }}
\end{gathered}
$$

\section{RESULTS AND DISCUSSION}

\section{Experimental results of the slit apertures}

Figures 5 and 6 show the experimental absorption coefficient results for the straight and tapered slit resonators, respectively, for lower and higher sound pressure levels generated via the loudspeaker.

In the case without bias flow, the tapered aperture exhibits a resonant frequency of approximately $460 \mathrm{~Hz}$. Conversely, the straight aperture exhibits a resonant frequency of approximately $340 \mathrm{~Hz}$. The higher resonant frequency obtained for the tapered aperture agrees with the theoretical prediction model developed by Tang [21] for circular apertures, which predicts a higher resonant frequency for the tapered circular aperture when compared to that of a circular straight aperture.

In both apertures, the resonant frequency is shifted higher when the bias flow increases.

In the no bias flow case, with a sound pressure level of $100 \mathrm{~dB}$, the straight aperture exhibits a narrow frequency range of acoustic energy absorption located around the resonant frequency with a high peak. Conversely, the tapered aperture exhibits a wider absorption frequency range with a reduced peak and an overall poor acoustic energy absorption. When the sound pressure level increases to $115 \mathrm{~dB}$, the straight aperture absorption still exhibits a narrow frequency range although an overall improvement of the absorption coefficient is obtained with a higher peak. The absorption coefficient of the tapered aperture when an SPL of $115 \mathrm{~dB}$ is applied also improves with a wider absorption range.

Introduction of the bias flow exhibits a tendency to widen the absorption range in the straight and tapered apertures, with a peak value reaching a maximum when the bias flow increases. A Further increase in the bias flow results in a reduced peak value. This behavior is similar to the results obtained by Wada and Ishii [11] and Tanaka et al. [13] for a liner with straight circular apertures.

As shown in Fig. 5, when the bias flow is applied, the sound pressure level has almost no effect on the absorption performance such that the curves for $100 \mathrm{~dB}$ and $115 \mathrm{~dB}$ exhibit almost identical values.

The peak value of the absorption coefficient for both the straight and tapered aperture is at its maximum when a bias flow Mach number of approximatly $9.71 \times 10^{-3}$ is applied.

According to Bechert [2], the maximum sound absorption caused by vorticity shedding is obtained if the Mach number of the bias flow at the aperture is approximately equal to the aperture area contraction ratio.

The area contraction ratio $(C R)$ is defined as the ratio of the area of the contracted jet passing through the aperture $\left(A_{\mathrm{Cjet}}\right)$ to the plate's area $\left(A_{\text {plate }}\right)$ as follows:

$$
C R=\frac{A_{\text {Cjet }}}{A_{\text {plate }}}=\sigma \frac{A_{\text {aperture }}}{A_{\text {plate }}}
$$

Wada and Ishii [11] conducted a simple parametric investigation of the jet contraction coefficient $\sigma$ and indicated that a value of 0.8 led to results close to the experimental results for a circular straight aperture with a Mach number bias flow velocity $M=1.19 \times 10^{-2}$.

In this study, the perforated plates area corresponds to $A_{\text {plate }}=7.85 \times 10^{-3} \mathrm{~m}^{2}$, and the apertures area corresponds to $A_{\text {aperture }}=10^{-4} \mathrm{~m}^{2}$.

For a $\sigma$ set to 0.8 , the peak values of the absorption coefficient in Figs. 5 and 6 are obtained for an area contraction ratio $C R=0.01$. It should be noted that the Mach number at the aperture corresponds to 0.0097 , and this value is close to the value of the area contraction ratio $C R$. This result is in good agreement with Bechert [2] theory to predict the optimum bias flow.

Fig. 5 shows one case with $95 \%$ confidence interval error bars as a reference. It is found that the error bars exhibit identical values in all of the cases.

\section{Numerical and analytical results of the slit apertures}

Absorption coefficient. Figure 5 compares results of the absorption coefficients obtained experimentally, numerically, and analytically for the straight slit aperture. Incident sound pressure levels of approximately $100 \mathrm{~dB}$ and $115 \mathrm{~dB}$ are considered. In the case without bias flow, at $100 \mathrm{~dB}$ SPL, the numerically and experimentally obtained results are in good agreement. At $350 \mathrm{~Hz}$, a $10 \%$ difference is observed. However, the analytical results are underestimated at frequencies lower than the resonant frequency. At higher frequencies, the results are in good agreement with the numerical and experimental results.

The numerical results also indicate that the absorption performance is affected by the sound pressure level of the sound source. A higher absorption coefficient at $115 \mathrm{~dB}$ is obtained when compared to the $100 \mathrm{~dB}$ case for frequencies equal to and lower than the resonant frequency. However, the results obtained at higher frequencies do not capture this improvement in the absorption performance.

When the bias flow of Mach number $M=9.71 \times 10^{-3}$ is applied, the numerical and analytical results are in good agreement with the experimental results.

Figure 6 shows a comparison of the absorption coefficients between results obtained experimentally and the results obtained via numerical simulations for the tapered slit aperture resonator. Incident sound pressure levels approximately corresponding to $100 \mathrm{~dB}$ and $115 \mathrm{~dB}$ are considered. In the case without bias flow, at $100 \mathrm{~dB}$ SPL, the numerically and experimentally obtained results are in good agreement.

The absorption coefficient results numerically obtained for the resonator are largely affected by the applied sound pressure level with a higher performance for the $115 \mathrm{~dB}$ sound pressure level source when compared to that of the $100 \mathrm{~dB}$ case. Additionally, the numerical and experimental results are in good agreement.

In the case of $M=9.71 \times 10^{-3}$ bias flow, the numerically obtained values are in good agreement with the experimental results for the frequencies around the resonant frequency. For frequencies away from the resonant frequency, the numerical results are overestimated compared to the experimental results although the 
trend is still well captured.

Flow field. All the flow field snapshots are obtained with respect to the instant where the area average velocity oscillation at the inlet of the aperture corresponds to a maximum. It should be noted that each period $T$ of the acoustic wave is divided into 20 steps.

Figures 7 and 8 show snapshots of the unsteady vorticity contour around the straight aperture in the case of sound pressure levels corresponding to $100 \mathrm{~dB}$ and $115 \mathrm{~dB}$, respectively. Both cases occur at an excitation frequency of $350 \mathrm{~Hz}$ which is close to the resonant frequency. For the $100 \mathrm{~dB}$ case, a shear layer is generated near the wall, although shed vortices are absent. Conversely, for the $115 \mathrm{~dB}$ case, in addition to the shear layer, larger vortices are created at the extremities of the aperture with higher vorticity magnitude. The existence of the vortices is the reason for the improvement of the calculated absorption coefficient when compared with the $100 \mathrm{~dB}$ case. Such improvement is due to the conversion of acoustic energy to vortical kinetic energy, which is subsequently dissipated via viscous dissipation.

Figures 9 and 10 show snapshots of the unsteady vorticity contour around the straight aperture in the case of sound pressure levels corresponding to $100 \mathrm{~dB}$ and $115 \mathrm{~dB}$, respectively. Both cases occur at an excitation frequency of $350 \mathrm{~Hz}$, with an applied bias flow of Mach number $M=9.71 \times 10^{-3}$. At $100 \mathrm{~dB}$, weak vortices embedded in the vorticity sheet are observed. At $115 \mathrm{~dB}$, stronger vortices are shed on the right side of the aperture. The absorption coefficient is not affected despite the clear difference in the flow field between the two cases. This shows that the bias flow forces the resonator response to the linear regime.

Figures 11 and 12 show snapshots of the unsteady vorticity contour around the tapered aperture in the case of sound pressure levels corresponding to $100 \mathrm{~dB}$ and $115 \mathrm{~dB}$. Both cases occur at an excitation frequency of $500 \mathrm{~Hz}$. With respect to the $100 \mathrm{~dB}$ case, a shear layer is generated near the wall with weak vorticity magnitude. The absence of vortices is the reason as to why the weak absorption coefficient is obtained in the experiment and in numerical simulation. With respect to the $115 \mathrm{~dB}$ case, in addition to the shear layer in the tapered aperture, the shear layer rolls up at the tip of the aperture creating vortices that are shed away. The shed vortices are the reason for the improvement in the absorption coefficient when compared to that in the $100 \mathrm{~dB}$ case. The sharp edge of the tapered aperture appears to aid in increasing the instability of the generated vortices, thereby resulting in their detachment from the aperture walls.

Figures 13 and 14 show snapshots of the unsteady vorticity contour around the tapered aperture in the case of sound pressure levels corresponding to $100 \mathrm{~dB}$ and $115 \mathrm{~dB}$, respectively. Both cases occur at an excitation frequency of $500 \mathrm{~Hz}$, with an applied bias flow of $M=9.71 \times 10^{-3}$. With respect to the $100 \mathrm{~dB}$ case, a vorticity sheet is observed on the right side of the aperture. A jet is formed and accompanied by a shear layer that is formed near the walls of the aperture, and the shear layer rolls up to create a continuous vorticity sheet. However, at $115 \mathrm{~dB}$, the shear layer creates discrete vortices of higher magnitude that are shed away. The absorption coefficient is not affected despite the clear difference in the flow field between the two cases.

Figures 15 and 16 show the time-averaged viscous dissipation (Eq. 12) for an acoustic wave with instantaneous streamlines around the straight and tapered apertures, respectively. The cases of a sound source of $115 \mathrm{~dB}$ and relatively high frequency of $700 \mathrm{~Hz}$ are chosen. The frequency is selected to understand the reason for the wider absorption frequency range obtained for the tapered aperture.

With respect to the straight aperture, viscous dissipation is caused by friction near the walls of the aperture with small vortices attached to the corners. With respect to the tapered aperture, in addition to the friction of walls, a large area of viscous dissipation is caused at the sharp edges of the aperture where vortices are gener- ated. These vortices hold the converted acoustic energy that is absorbed via viscous dissipation.

Time-averaged viscous dissipation integrated over the calculation domain (Eq. 14) in Figs. 15 and 16, for the tapered aperture is approximately 2.8 times that for the straight aperture. The absorption coefficient for the tapered aperture is also approximately 2.8 times that of the straight aperture.

With respect to the $115 \mathrm{~dB}$ SPL case for the straight aperture, the underestimation of the absorption coefficient at higher frequencies in the numerical simulation when compared to the experimental results is potentially attributed to the existence of vortices around the aperture in the experiment, thereby leading to the nonlinear response of the resonator. As shown in Fig 15, vortices are absent in the simulation and thus the resonator response is in the linear regime.

\section{CONCLUSION}

In this study, experimental results of the acoustic behavior of two different slit apertures (i.e., straight and tapered apertures with and without bias flow) were obtained via an impedance tube test rig.

In the case without bias flow, straight aperture results in a narrow absorption frequency range centered around the resonant frequency. Conversely, the tapered aperture tends to exhibit a wider absorption frequency range. Additionally, the tapered aperture has a higher resonant frequency than the straight aperture.

For both plates (i.e., straight and tapered apertures), the introduction of the bias flow increases the peak absorption coefficient. For the plates tested, the peak is obtained for an applied bias flow of Mach number approximately $M=9.71 \times 10^{-3}$. Furthermore, the bias flow results in a wider absorption frequency range. Moreover, an increase in the bias flow increases the resonant frequency.

Large eddy simulations were conducted in conditions approximately similar to that of experiments for validation purposes of the acoustic behavior of the resonators, and also to visualize the flow field around the apertures. The absorption coefficients obtained by CAA agree well with the experimental results.

The study also discusses the flow field around the apertures and effect of flow structures around the apertures on the absorption performance of the resonators.

The results indicate that the presence of vortex shedding increases the absorption coefficient. Increases in the sound pressure level intensifies the vortex shedding, thereby leading to a better absorption performance than that for lower sound pressure levels. Additionally, the introduction of a bias flow (which is a method to facilitate the vortex shedding even for lower sound pressure levels) improves the absorption performance.

\section{ACKNOWLEDGMENTS}

The numerical simulations were conducted at the Supercomputer Center in Kyushu University.

\section{REFERENCES}

[1] Howe, M.S., 1979, "Attenuation of sound in a low Mach number nozzle flow," Journal of Fluid Mechanics, 91, pp. 209-229.

[2] Bechert, D.W., 1980, "Sound Absorption Caused by Vorticity Shedding, Demonstrated with a Jet Flow," Journal of Sound and Vibration, 70(3), pp. 389-405.

[3] Hughes, I.J., and Dowling, A.P., 1990, "The absorption of sound by perforated linings," Journal of Fluid Mechanics, 218, pp. 299-335.

[4] Lahiri, C., Enghardt, L., Bake, F., Sadig, S., and Gerendas, M., 2011, "Establishment of a high-quality database for the modelling of perforated liners, Journal of Engineering for Gas Turbines and Power, 133(9):091503 (9 pages)

[5] Zhao, D., and Li, X. Y., 2015, “A review of acoustic dampers applied to combustion chambers in aerospace industry," Progress in Aerospace Science, 74, pp. 114-130.

[6] Mendez, S. and Eldredge, J.D., 2009, “Acoustic modeling of 
perforated plates with bias flow for Large-Eddy Simulations," Journal of Computational Physics, 228, pp. 4757-4772.

[7] Ji, C., and Zhao, D., 2014, "Two-dimensional lattice Boltzmann investigation of sound absorption of perforated orifices with different geometric shapes," Aerospace Science and Technology, 39, pp. 40-47.

[8] Roche, J. M., Leylekian, L., Delattre, G., and Vuillot, F., 2009 "Aircraft fan noise absorption: DNS of the acoustic dissipation of resonant liners," Proceedings of 15th AIAA/CEAS Aeroacoustics Conference, Paper No. AIAA 2009-3146, pp. 0116.

[9] Ramdani, S., Yamasaki, N., Inokuchi, Y., and Ishii, T., 2017, "Large eddy simulation of conventional and bias flow acoustic liners," ASME Turbo Expo, Volume 2C, pp V02CT43A007 (16 pages).

[10] Tam, C.K.W., Ju, H., Jones, M.G., Watson, W.R., and Parrott, T.L., 2005, "A computational and experimental study of slit resonators," Journal of Sound and Vibration, 284, pp. $947-$ 984.

[11] Wada, K., and Ishii, T., 2013, "Acoustic absorption of perforated plates with fine jets: experimental results and analytical models," Proceedings of 4th International Conference on Jets, Wakes and Separated Flows, Paper No. ICJWSF20131 127.eda

[12] Luong, T., Howe, M.S., and McGowan, R.S., 2005, "On the Rayleigh conductivity of a bias-flow aperture," Journal of Fluids and Structures, 21, pp. 769-778.

[13] Tanaka, Y., Yamasaki, N., Inokuchi, Y., and Ishii, T., 2015,
"Effects of aperture geometry on impedance of active acoustic liners," Proceedings of International Gas Turbine Conference, ISBN978-4-89111-008-6, pp. 1052-1058.

[14] International Standard Organization, 1998, “Acoustics - Determination of sound absorption coefficient and impedance in impedance tubes - Part 2: Transfer-function method," ISO 10534-2.

[15] Tam, C.K.W., and Kurbatskii, K.A., 2000, "Microfluid dynamics and acoustics of resonant liners," AIAA Journal, 38(8), pp. 1331-1339.

[16] Zhang, Q., and Bodony, D.J., 2011, "Numerical simulation of two-dimensional acoustic liners with high-speed grazing flow," AIAA Journal, 49(2), pp. 365-382.

[17] Greitzer, E.M., Tan, C.S., and Graf, M.B., 2004, Internal Flow-Concepts and Applications, Cambridge University Press, pp. 94-103.

[18] Ulf, R. K., Tor, E. V., 1994, "On the design of resonant absorbers using a slotted plate," Applied Acoustics Journal, 43, pp. 39-48.

[19] Hughes, I.J., Dowling, A.P., 1992, "Sound absorption by a screen with a regular array of slits," Journal of Sound and Vibration, 156, pp. 387-405.

[20] Jing, X., Sun, X., 2000, "Effect of plate thickness on impedance of perforated plates with bias flow," AIAA Journal, 38(9), pp. 1573-1578.

[21] Tang, S.K., 2005, "On Helmholtz resonators with tapered necks," Journal of Sound and Vibration, 279, pp. 1085-1096.

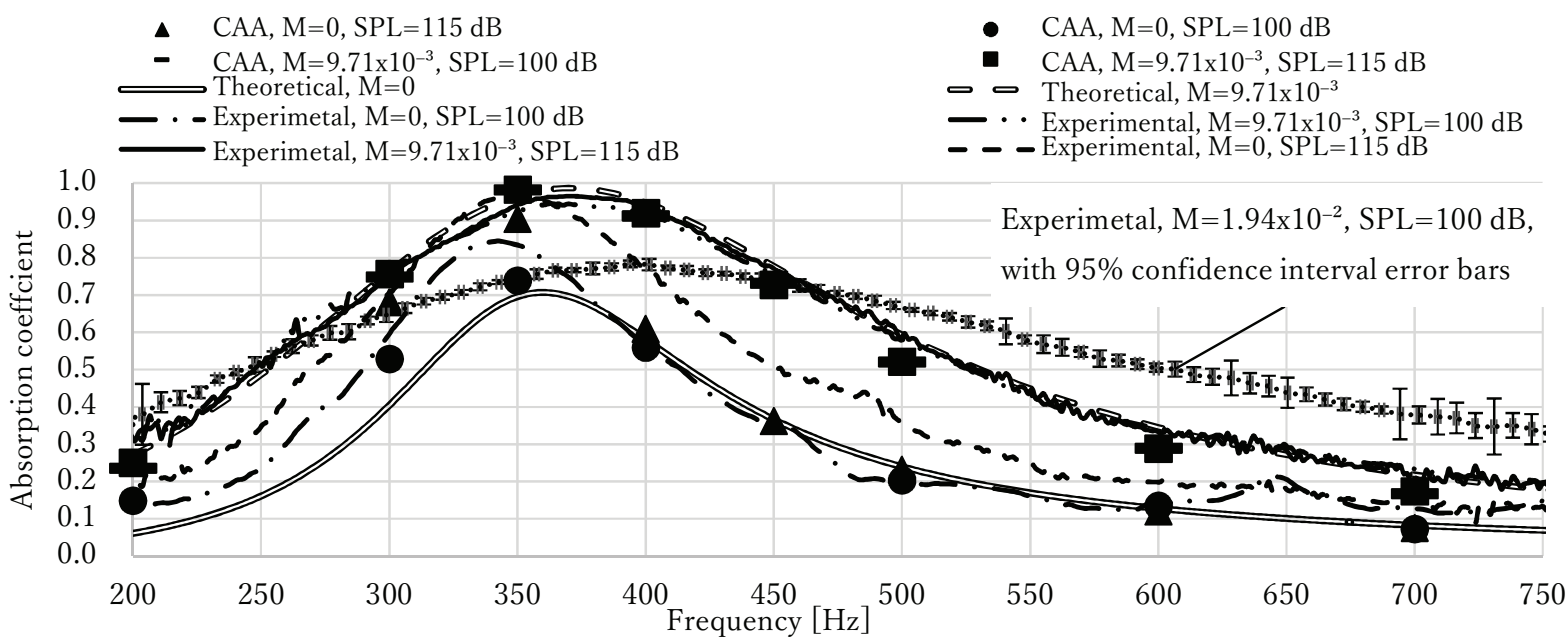

Fig. 5 Absorption coefficients for the straight slit aperture

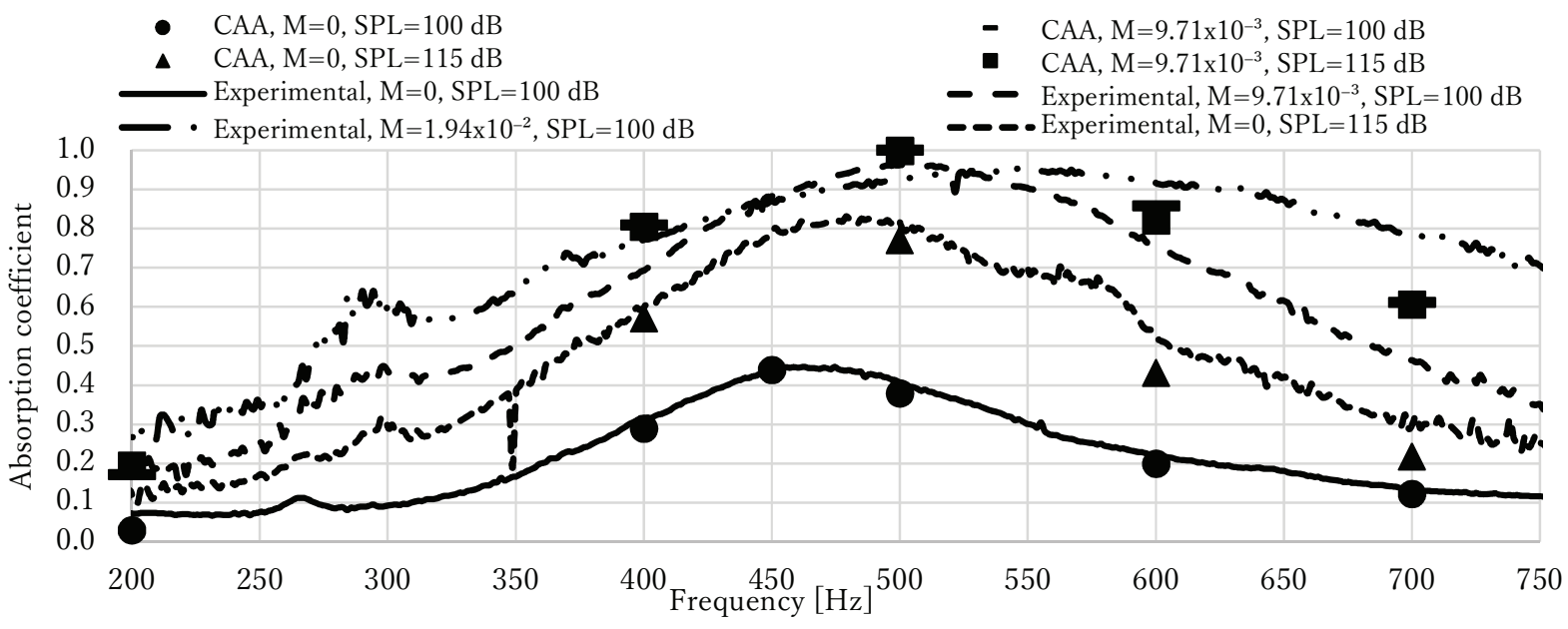

Fig. 6 Absorption coefficients for the tapered slit aperture 


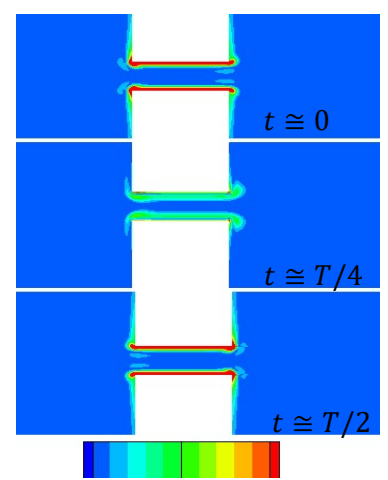

0.0

Fig. 7 Non-dimensional vorticity magnitude, straight aperture, $350 \mathrm{~Hz}$ and $100 \mathrm{~dB}$ without bias flow
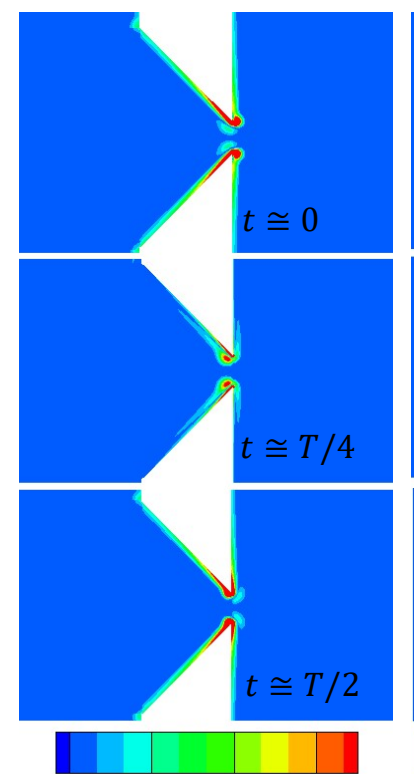

0.0

0.005

Fig. 11 Non-dimensional vorticity, tapered aperture, 500

$\mathrm{Hz}$ and $100 \mathrm{~dB}$ without bias flow

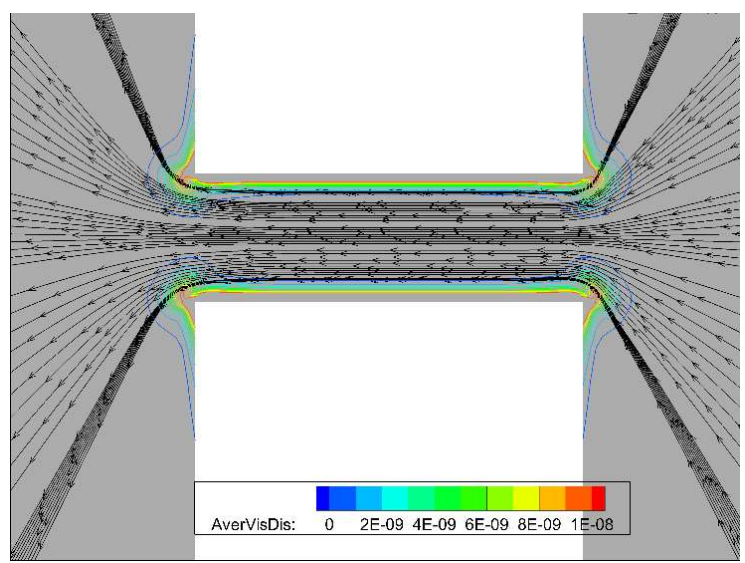

Fig. 15 Time-averaged viscous dissipation, straight aperture, without bias flow $700 \mathrm{~Hz}, 115 \mathrm{~dB} t \cong T / 2$

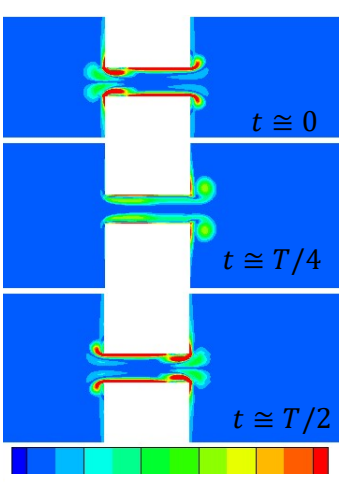

0.0

0.05

Non-dimensional vorticity magnitude, straight aperture, 350 $\mathrm{Hz}$ and $115 \mathrm{~dB}$ without bias flow
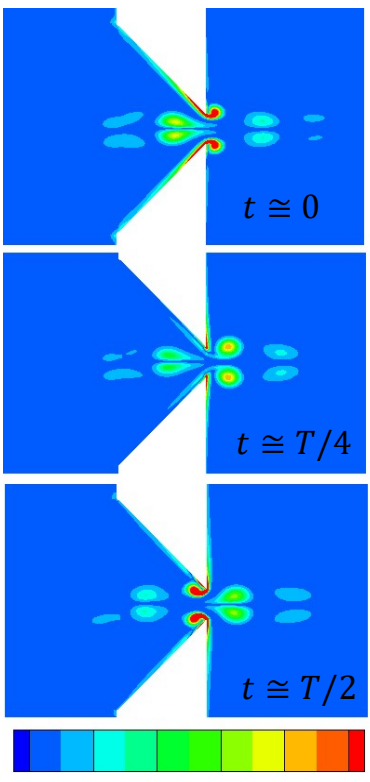

$0.00 \quad 0.05$

Fig. 12 Non-dimensional vorticity, tapered aperture, 500 $\mathrm{Hz}$ and $115 \mathrm{~dB}$ without bias flow

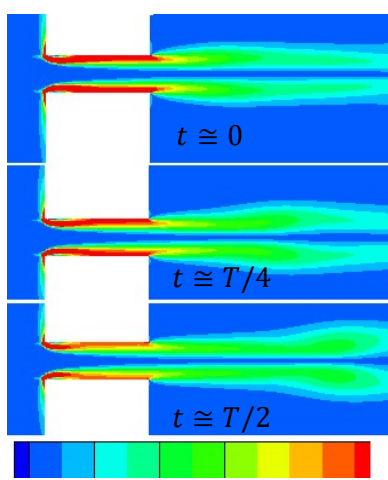

0.00

Fig 9 Non-dimensional vort magnitude, straight aperture $\mathrm{M}=9.71 \times \mathbf{1 0}^{-\mathbf{3}}, 350 \mathrm{~Hz}$ and 100 $\mathrm{dB}$

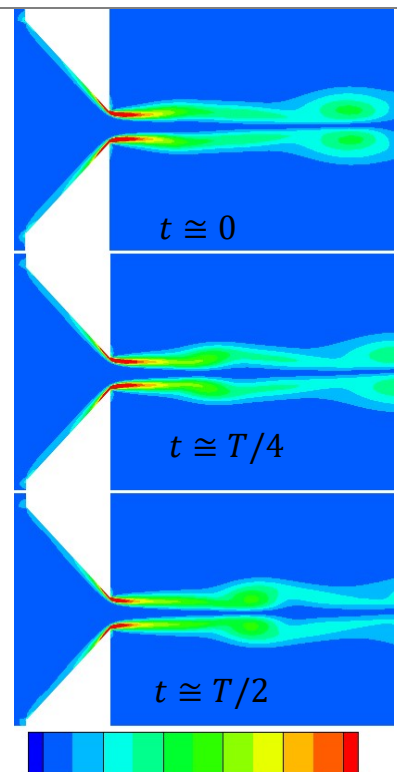

$0.00 \quad 0.05$

Fig. 13 Non-dimensional vorticity magnitude, tapered aperture, $\mathrm{M}=9.71 \times 10^{-3}, 500 \mathrm{~Hz}$ and $115 \mathrm{~dB}$

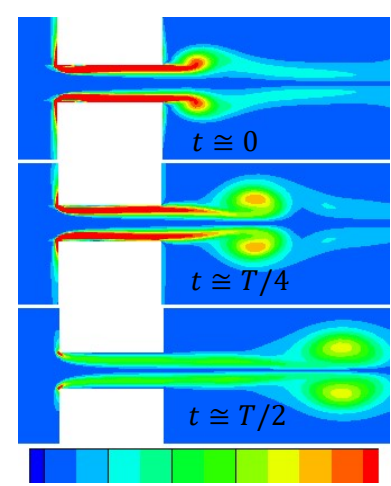

0.00

0.05

Fig. 10 Non-dimensional vorticity magnitude, straight aperture, $\mathrm{M}=9.71 \times \mathbf{1 0}^{\mathbf{- 3}}$, $350 \mathrm{~Hz}$ and $115 \mathrm{~dB}$

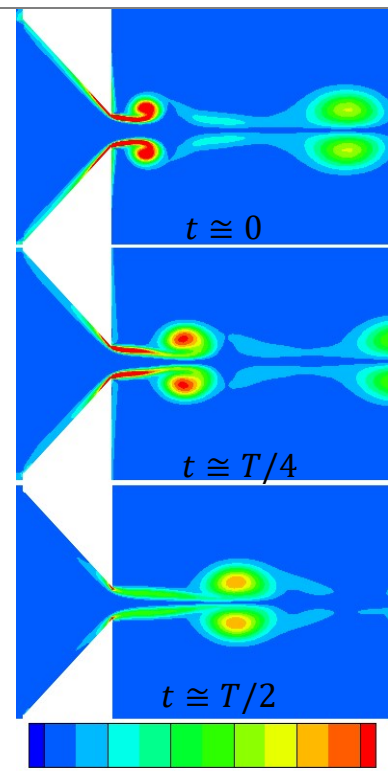

0.00

0.05

Fig. 14 Non-dimensional vorticity magnitude, tapered aperture, $M=9.71 \times 10^{-3}, 500$ $\mathrm{Hz}$ and $100 \mathrm{~dB}$

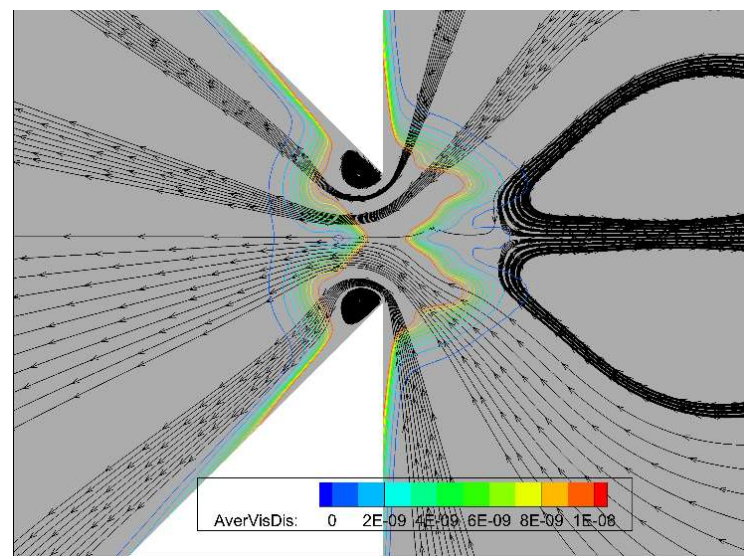

Fig. 16 Time-averaged viscous dissipation, tapered aperture, without bias flow $700 \mathrm{~Hz}, 115 \mathrm{~dB}, t \cong T / 2$ 